Trauma Berufskrankh 2009 · 11 [Suppl 2]: 187-192

DOI 10.1007/s10039-009-1472-7

Online publiziert: 10. Mai 2009

(c) Springer Medizin Verlag 2009

\author{
G. Walter · W. Hirschberger - R. Hoffmann \\ BG-Unfallklinik Frankfurt am Main
}

\section{Mikrobiologie bei Osteitis}

\section{Evidenzbasierte Medizin}

Die Entnahme von Gewebeproben zur mikrobiologischen Aufarbeitung gilt heute als „Goldstandard“ in der Diagnostik und Therapie der Osteitis. Eine gezielte Therapie mit Antibiotika, neben der unabdingbar notwendigen chirurgischen Therapie, ist nur in Kenntnis des vorhandenen Keimspektrums und der Erregerresistenzen möglich. Es stellt sich jedoch die Frage, auf welche wissenschaftliche Basis dieser allgemein akzeptierte Goldstandard gründet und wie hoch seine Aussagekraft einzuschätzen ist. Der vorliegende Beitrag gibt einen Überblick.

\section{Evidenzbasierte Medizin (EbM)}

EbM bezeichnet jede Form von medizinischen Maßnahmen, bei der patientenorientierte Entscheidungen ausdrücklich auf der Grundlage von nachgewiesenen Wirksamkeiten getroffen werden. Der Wirksamkeitsnachweis hierbei erfolgt durch statistische Verfahren.

\section{Historie}

In Großbritannien wurde eine der ersten kontrollierten Studien durch James Lind im Jahre 1753 veröffentlicht ( $\bullet$ Abb. 1). Er therapierte damals an Skorbut erkrankte Patienten mit Zitronen und Orangen und dokumentierte den Heilverlauf.

Die Idee, in der medizinischen Forschung eine „systematische klinische Beobachtung " einzuführen, wurde im deutschsprachigen Raum erstmals 1848 durch den im Wien tätigen ungarischen Arzt Ignaz Semmelweis formuliert. Im englischsprachigen Raum dagegen wurde bereits 1793 von dem schottischen Arzt George Fordyce ein Artikel mit dem Titel
"An attempt to improve the evidence of medicine" veröffentlicht.

Den Beginn der aktuellen Bemühungen um evidenzbasierte Medizin markiert das 1972 veröffentlichte Buch" Effectiveness and efficiency: random reflections on health services" des britischen Epidemiologen Prof. A. Cochrane [6]. Das heute international arbeitende Netzwerk von Wissenschaftlern zur Wirksamkeitsbewertung von Studien in der Medizin (Cochrane Collaboration) wurde in Anerkennung seiner Bemühungen nach ihm benannt.

Der Begriff evidenzbasierte Medizin (EbM) wurde Anfang der 1990er Jahre von Gordon Guyatt aus der Gruppe um David Sackett an der McMaster University, Ha-

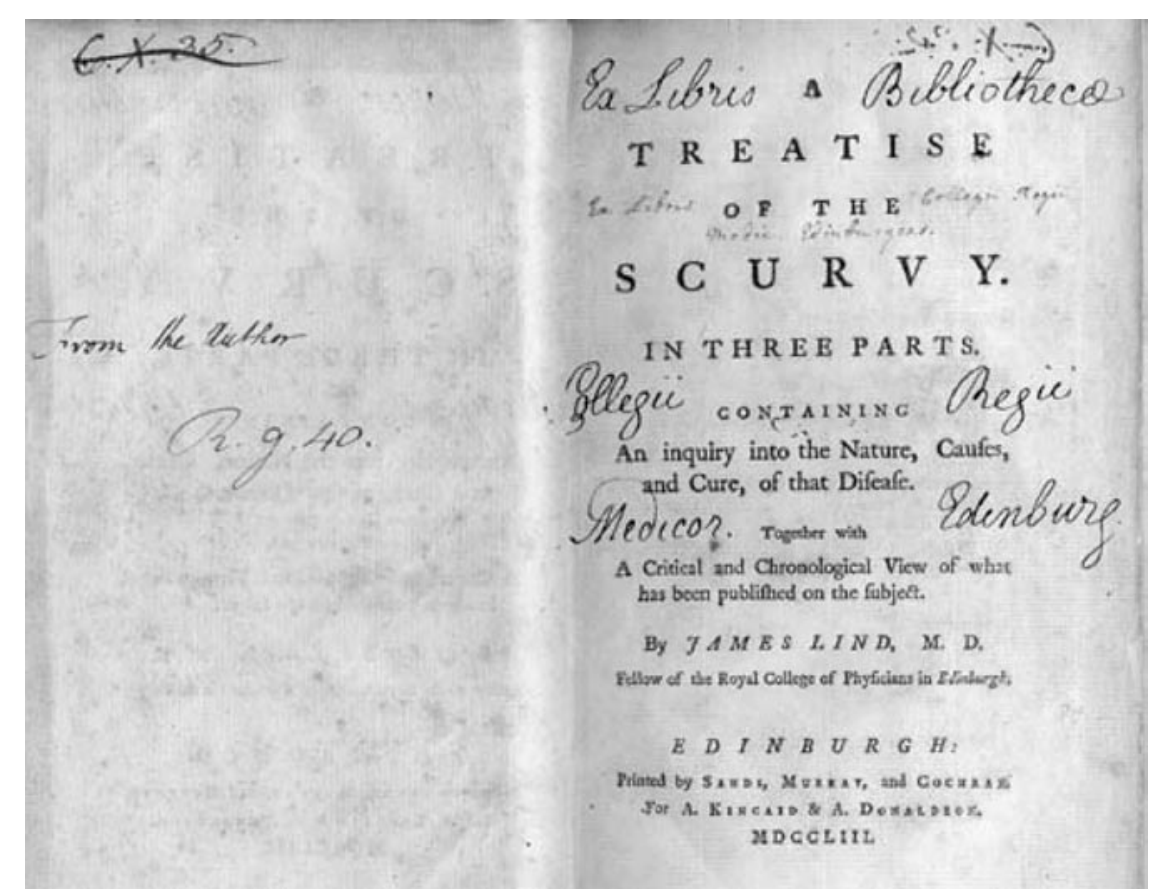

Abb. 1 \ Titelblatt der 1753 von James Lind veröffentlichten Untersuchungen zur Skorbutbehandlung milton, Kanada eingeführt. Im deutschsprachigen Raum erschienen die ersten Publikationen zu diesem Thema Mitte der 1990er Jahre. Sie fielen in einer Zeit zunehmend knapper werdender Ressourcen im Gesundheitswesen auf fruchtbaren Boden. Man hoffte, durch sorgfältige wissenschaftliche Evaluation Therapiestrategien zu identifizieren, welche effektiv und damit auch kostengünstig einzusetzen sind. Die weniger oder nicht wirksamen Verfahren sollten aufgespürt und von einer Kostenerstattung ausgeschlossen werden [20]. Die Verbesserung der medizinischen Versorgung und die Identifizierung von medizinischen Teilgebieten, in denen noch eine verstärkte Forschungstätigkeit nötig ist, waren nun nicht mehr 
Klinische Studien (randomisiert, kontrolliert, prospektiv) Epidemiologische Studien (retrospektiv) Systematische Übersichtsarbeiten

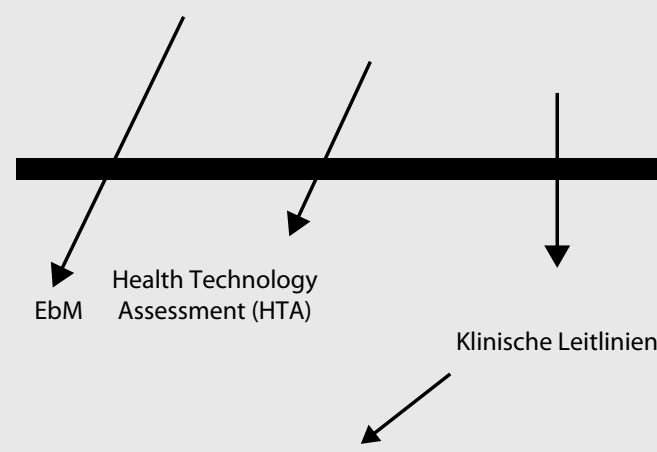

Strukturierte Behandlungsprogramme

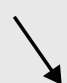

Klinische Behandlungspfade

Abb. $2 \Delta$ Wissenstransfer von der Forschung in die Praxis. (Mod. nach [1])

alleiniges Ziel der wissenschaftlichen Anstrengungen. Um dies zu erreichen, mussten die Flut der wissenschaftlichen Arbeiten aus den verschiedenen Fachbereichen gesichtet und mit einem eindeutigen Bewertungsmaßstab versehen werden, woraus sich dann der Grad der Evidenz für die einzelnen Studien ergibt [19].

\section{Begriffsdefinitionen}

Leider gib es bis heute keine einheitliche und allgemein anerkannte Bewertungsgrundlage zur Einteilung der Evidenzstufen und Empfehlungsgrade. Die nationalen und internationalen Arbeitsgruppen und Fachgesellschaften verwenden unterschiedliche Bewertungsmaßstäbe. Die in $\bullet$ Tab. 1 aufgeführten Grundlagen der Klassifizierung der Evidenz sind jedoch vergleichbar.

Die Evidenzstärke ist vom Empfehlungsgrad (Härtegrad) zu unterscheiden. Dieser stützt sich auf die medizinische Erfahrung. So kann es vorkommen, dass Studien mit sehr hohem Evidenzgrad einen sehr niedrigen Empfehlungsgrad aufweisen (z. B. tierexperimentell nachgewiesener Vorteil einer Therapiemethode, die am Menschen noch nicht erprobt wurde). Umgekehrt ist es möglich, dass ein therapeutisches Vorgehen mit einem hohen Empfehlungsgrad versehen ist bei gleichzeitig sehr niedriger Evidenz (z. B. Reanimation bei Herzstillstand) [16].

Beide genannten Begriffe sind vom so genannten „Goldstandard“ zu unterscheiden. Mit Goldstandard wird ein Verfahren in Diagnostik oder Therapie bezeich- net, das bislang unübertroffen scheint und als Maßstab gelten kann. Meist handelt es sich dabei um ein allgemein anerkanntes Verfahren, das bereits seit längerer Zeit von verschiedenen Untersuchern angewandt wird. Eine statistische Wirksamkeitsüberprüfung des betreffenden Verfahrens muss nicht unbedingt stattgefunden haben.

Im Rahmen der Bemühungen um mehr Evidenz in der Medizin und den hieraus gewonnenen Erkenntnissen entwickelte die Arbeitsgemeinschaft der Wissenschaftlichen Medizinischen Fachgesellschaften e.V. (AWMF) Leitlinien für Diagnostik und Therapie.

\section{EbM in der wissenschaftlichen Diskussion}

Nach Einführung von EbM und der AWMF-Leitlinien fanden sich ablehnende und kritische, aber auch viele positive Stimmen. Es folgte eine Phase der kontrovers und zu Teilen heftig geführten (nicht immer sachlichen) und von Sachverstand geprägten Diskussion.

„Evidenzbasierte Medizin in der Chirurgie mutet an wie ein Partygespräch über ein Buch, das allenfalls einer wirklich gelesen hat und von dem mancher den Klappentext, einige die Kritiken und viele nur den Titel kennen“. [15]

Die Befürworter der EbM weisen darauf hin, dass nun Riten und Mythen aus der Medizin verschwinden würden. Eine interessengeprägte Meinungsbildung hätte weniger Aussicht auf Erfolg, da sich wissenschaftliche Ergebnisse einer Über- prüfung unterziehen müssen. Die Bewertung der klinischen Erfahrung würde mit Einführung der EbM auf das notwendige und sinnvolle Maß reduziert. Eine „eminenzbasierte Medizin“ sei so zum Scheitern verurteilt. Weitere Argumente für eine EbM sind die rationale Absicherung von neuen Verfahren vor deren generellen Einsatz. Auch verspricht man sich durch EbM einen durchschaubareren, vollständigeren und schnelleren Transfer von Forschungsergebnissen in die Praxis. Dies scheint bei einer immer kürzeren Halbwertszeit des medizinischen Wissens dringend geboten. Die ständig wachsende Informationsmenge verlangt effiziente Strategien, um unwichtige von wertvollen Daten zu trennen. Letztere müssen auf ihre Qualität hin überprüft und für die klinische Praxis verfügbar gemacht werden $[2,7]$. Das Konzept, systematische Übersichtsarbeiten und Metaanalysen zu erstellen, scheint sich hierbei zu bewähren $[17,19]$.

Die Aussagekraft der so erstellten Übersichtsarbeiten und Metaanalysen hängt in erheblichem Maße von der Qualität sowohl der Originalarbeiten als auch des Auswahlverfahrens der in die Analyse aufgenommenen Originalarbeiten ab. Sowohl die Originalarbeiten als auch die erstellten systematischen Übersichtsarbeiten oder Metaanalysen können erhebliche methodische Schwächen aufweisen $[8,10]$. Schätzungsweise kommen heute nur etwa 50\% des wissenschaftlich erarbeiteten Wissens zur Veröffentlichung, die Hälfte geht nicht berichtet verloren (- Abb. 2).

Kritiker der EbM sehen die ärztliche Therapiefreiheit in erheblichem Maße bedroht. Es wird von unkritischer, kostenoptimierter, nicht mehr individueller „Kochbuchmedizin“ gesprochen. Man weist darauf hin, dass Ergebnisse aus großen Studien z. T. für die Therapie des Individuums unbrauchbar sind. Eine Beweisführung ist aufgrund kleiner Fallzahlen und ethisch nicht vertretbarer Studien in vielen Bereichen der Medizin nur schwer oder gar nicht möglich. Das Fehlen von bewiesenem Nutzen ist jedoch nicht mit dem Fehlen von Nutzen gleichzusetzen.

Aus diesem Blickwinkel handelt es sich bei der Einführung von Leitlinien für Diagnostik und Therapie vieler Erkran- 
Tab. 1 Grundlagen zur Klassifizierung der Evidenz

\begin{tabular}{ll} 
Evidenzlevel & Studie \\
I & $\begin{array}{l}\text { Randomisiert-kontrollierte } \\
\text { Studien }\end{array}$ \\
II & Kohortenstudien \\
III & Fall-Kontroll-Studien \\
IV & Fallserien \\
\hline V & Expertenmeinung \\
\hline
\end{tabular}

kungen um ein zweischneidiges Schwert. Einerseits werden dem behandelnden Arzt wichtige Informationen zur Verfügung gestellt, andererseits wird bei Abweichungen von den vorgegebenen Therapie- und Diagnostikstandards eine zunehmende juristische Angreifbarkeit befürchtet. Es erscheinen gerade im Falle einer individuellen Medizin, unter Abweichung von Therapiestandards, eine gute Aufklärung des Patienten und eine saubere Dokumentation der Therapie notwendig. Die klinische Erfahrung des behandelnden Arztes und das Wissen um die Bedürfnisse des einzelnen Patienten erscheinen in diesem Therapiekonzept unterbewertet.

In einer Arbeit aus dem Jahre 1996 begegneten Sackett et al. [18] den oben genannten Einwänden mit einer knappen, aber dennoch umfassenden Beschreibung, was EbM in der täglichen Praxis leisten soll.

„EbM ist der gewissenhafte, ausdrückliche und vernünftige Gebrauch der gegenwärtig besten externen Evidenz für Entscheidungen in der medizinischen Versorgung individueller Patienten. Die Praxis der EbM bedeutet die Integration individueller klinischer Expertise mit der besten verfügbaren externen Evidenz aus systematischer Forschung. “ [18]

\section{Material}

Die Literatursuche für diese Arbeit erfolgte auf elektronischem Weg. Nach Durchsicht der einschlägigen Datenbanken (The Cochrane Library, Medline und Google) zeigte sich jedoch schnell, dass zum Thema Mikrobiologie bei Osteitis keine Studien vorhanden sind. Es fanden sich zwar Einträge zur Antibiotikatherapie der hämatogenen Osteitis, Arbeiten über das eigentliche Thema konnten jedoch nicht entdeckt werden.

Trauma Berufskrankh 2009 · 11 [Suppl 2]: 187-192 DOI 10.1007/s10039-009-1472-7

(c) Springer Medizin Verlag 2009

\section{G. Walter $\cdot$ W. Hirschberger $\cdot$ R. Hoffmann \\ Mikrobiologie bei Osteitis. Evidenzbasierte Medizin}

\section{Zusammenfassung}

Der vorliegende Beitrag befasst sich mit der Frage, auf welcher wissenschaftlichen Basis der allgemein akzeptierte Goldstandard in der Diagnostik und Therapie der Osteitis - die Entnahme von Gewebeproben zur mikrobiologischen Aufarbeitung - gründet und wie hoch seine Aussagekraft ist. Zu periprothetischer Infektion/Osteitis finden sich derzeit in der Literatur nur Level-III- und -IV-Studien, Level-I- und -II-Studien fehlen. Die Diagnose eines frühen, akuten periprothetischen Infektes/einer akuten Osteitis hängt von unterschiedlichen Kriterien und Definitionen ab, ist in der Regel jedoch rasch zu stellen. Schwierigkeiten ergeben sich bei chronischen Infekten. Eine Sicherung der Diagnose durch eine Punktion zur Erregerbestimmung ist häufig nicht möglich, das Punktat sollte des- halb neben der Anlage einer Kultur zytologisch mittels Zellzählung und -differenzierung untersucht werden. Als zusätzliche Methode zur Diagnosesicherung kommen die arthroskopische oder offene Biopsie in Frage. In Abhängigkeit von den Befundergebnissen, der Dauer der Implantatlage und dem Allgemeinzustand des Patienten wird eine individuelle Behandlung eingeleitet. Dringend erforderlich sind einheitliche Definitionen der Schlüsselbegriffe in der septischen Chirurgie. Einen ersten Schritt stellt die Gründung der Arbeitsgemeinschaft Septische Chirurgie dar.

\section{Schlüsselwörter}

Akute/chronische Osteitis - Gelenkpunktion . Individuelle Behandlung · Belastbare

Evidenz - Arbeitskreis septische Chirurgie

\section{Microbiology in osteomyelitis. Evidence-based medicine}

\section{Abstract}

The present article discusses the scientific principles and reliability of the "gold standards" in osteomyelitis diagnosis and microbiological methods of tissue biopsies. Currently, only Level III and IV studies can be found in the literature, while Level I and II studies are lacking. Diagnosis of early and acute periprosthetic infection/acute osteomyelitis is usually straightforward; problems arise in the case of chronic infection. Reliable diagnosis by means of joint puncture to identify the pathogen is not possible; in addition to cell culture, the aspirate should be further analysed cytologically using cell count- ing and differentiation. Additional diagnostic methods include arthroscopic synovial biopsy. Regardless of the findings, duration of the implant's position, and the patient's general condition, individual therapy is initiated. The lack of robust evidence in septic surgery needs to be rectified urgently; the formation of the working group on septic surgery represents a first step in this direction.

\section{Keywords}

Acute/chronic osteomyelitis - Joint puncture . Individual therapy · Robust evidence . Working group on septic surgery 
Unter dem Gesichtspunkt, dass jeder periprothetische Infekt eine spezielle Form der Osteitis darstellt, wurde die Literatursuche entsprechend ausgedehnt. Dadurch konnten einige zum erweiterten Thema passende Arbeiten identifiziert werden. Nach deren Durchsicht zeigte sich jedoch, dass lediglich Level-III- und -IV-Studien vorhanden sind. Studien, die den EbM-Kriterien für eine Level-I- und II-Einstufung entsprechen, fehlen.

Die im Folgenden stichpunktartig aufgeführten Themen sind abschließend nicht geklärt:

\section{Diagnostik der Osteitis.}

- Einheitliche Definition der Osteitis

- Anzahl der nötigen Gewebeproben zur sicheren mikrobiologischen Diagnostik einer Osteitis

- Einfluss einer laufenden systemischen Antibiotikagabe auf das mikrobiologische Untersuchungsergebnis einer Gewebeprobe oder eines Punktates

- Stellenwert der Histologie in der Infektdiagnostik

- Stellenwert eines positiven Keimnachweises bei sonst unauffälliger Klinik (Kontamination/Infektion).

\section{Therapie der Osteitis.}

- Dauer der notwendigen systemischen Antibiotikatherapie der Osteitis oder des periprothetischen Infektes

- Stellenwert der lokalen Antibiotikaapplikation in der Osteitistherapie

- Nutzen eines lokalen Platzhalters nach Prothesenexplantation

- Ein- oder zweizeitiger Prothesenwechsel.

\section{Ergebnisse}

\section{Diagnose}

Im klinischen Alltag ist die Diagnosestellung eines frühen und akuten periprothetischen Infektes oder einer akuten Osteitis in aller Regel unproblematisch. Das klinische Bild und der laborchemische Verlauf sind meist eindeutig. Im Unterschied hierzu bereitet die Diagnostik des chronischen "Low-Grade-Infektes“ häufig Probleme. Diese Infektionen sind symptomarm und nur schwer von aseptischen entzündlichen Reaktionen oder Prothesenlockerungen zu unterscheiden.

Zur Diagnostik des periprothetischen Infektes (Osteitis) ist die Punktion der betreffenden Region zur Gewinnung von Material zur mikrobiologischen Aufarbeitung als Goldstandard anerkannt. Sie gilt als wichtigste diagnostische Maßnahme [14]. Aber trotz Einhaltung der drei wesentlichen Regeln:

- Rascher Transport ins mikrobiologische Labor

- Punktion unter Operationsbedingungen (Sterilität)

- Bebrütungsdauer von 14 Tagen.

gelingt nur in etwa 65\% der Punktionen ein Keimnachweis durch Kultur [3, 14]. Auch in großen Studien mit mehr als 2000 untersuchten Hüfttotalendoprothesen konnten nur $82 \%$ der Infektionen durch eine präoperative Punktion aufgedeckt werden [21]. Etwa jeder 5. periprothetische Infekt bleibt somit durch eine präoperative Punktion zur klassischen mikrobiologischen Diagnostik undiagnostiziert.

In der Zusammenschau der zur Verfügung stehenden Literatur finden sich für den Aussagewert einer präoperativen Gelenkpunktion zur Erregerbestimmung sehr unterschiedliche Angaben, zur Sensitivität von 45-100\%, zur Spezifität von 67$100 \%[9,11,12,24]$. Umgekehrt müssen jedoch auch positive Punktionsbefunde kritisch bewertet werden, da sie durch Kontamination bei der Probenentnahme oder im Labor zustande kommen können.

Von einer sicheren Diagnosestellung durch eine Gelenkpunktion zur Erregerbestimmung kann somit nicht die Rede sein. Zur Diagnosesicherung sollte das durch Punktion gewonnene Material neben der Anlage einer Kultur weiter untersucht werden. Bewährt hat sich dabei die Methode der zytologischen Untersuchung mittels Zellzählung und -differenzierung $[13,22]$.

Die Gramfärbung von Gelenkpunktaten und Gewebeproben besitzt für die Infektdiagnostik aufgrund der geringen Sensitivität keine wesentliche Bedeutung $[3,4]$.

Molekularbiologische Untersuchungen der Synovialflüssigkeit (PCR) weisen ebenfalls eine Vielzahl falsch-positiver Ergebnisse auf und sind mit dem Nach- teil behaftet, dass über die Antibiotikaresistenz des nachgewiesenen Keims keine Aussage getroffen werden kann.

Als weiterführende Methode zur Diagnoseabsicherung scheint sich die arthroskopische Biopsie der Synovia bewährt zu haben. Fuerst et al. [12] konnten in einer Arbeit aus dem Jahr 2005 für dieses Verfahren eine Sensitivität von $100 \%$ und eine Spezifität von 94,7\% nachweisen.

\section{Eigene Ergebnisse}

\section{Vorgehen}

In der eigenen Klinik hat sich das im Folgenden beschriebene diagnostische und therapeutische Vorgehen bewährt.

Den höchsten Stellenwert in der Diagnostik besitzt der klinische Befund, von welchem das weitere diagnostische Vorgehen abhängt. Die im Rahmen der Diagnostik gewonnenen Befunde ordnen sich in der Wertigkeit dem führenden klinischen Befund unter.

Zur Basisdiagnostik gehören:

- die Sonographie,

- die Röntgendiagnostik,

- die Bestimmung der Infektparameter [Blutbild und CRP (C-reaktives Protein)] und

- die Punktion unter diagnostischen Gesichtspunkten.

Die Punktion erfolgt unter Operationsbedingungen und radiologischer Kontrolle. Dabei sollte, wenn dies klinisch vertretbar ist, eine laufende systemische Therapie mit Antibiotika 5-7 Tage vor der Punktion abgesetzt werden. Das so gewonnene Material wird zur mikrobiologischen und laborchemischen Aufarbeitung eingeschickt.

Bei Fehlen der klassischen Infektzeichen, aber persistierender Klinik und negativem Punktionsbefund wird ggf. eine erweiterte Diagnostik notwendig. Einsatz finden hier die Magnetresonanztomographie (MRT) und die Szintigraphie. In seltenen Fällen kommt die ${ }^{18} \mathrm{~F}$-Fluordesoxyglukose-Positronenemissionstomographie $\left({ }^{18} \mathrm{~F}-\mathrm{FDG}-\mathrm{PET}\right)$ zur Anwendung, und es wird evtl. eine Probefreilegung notwendig.

In Abhängigkeit von den Befundergebnissen, der Dauer der Implantatlage und dem Allgemeinzustand des Patienten wird 

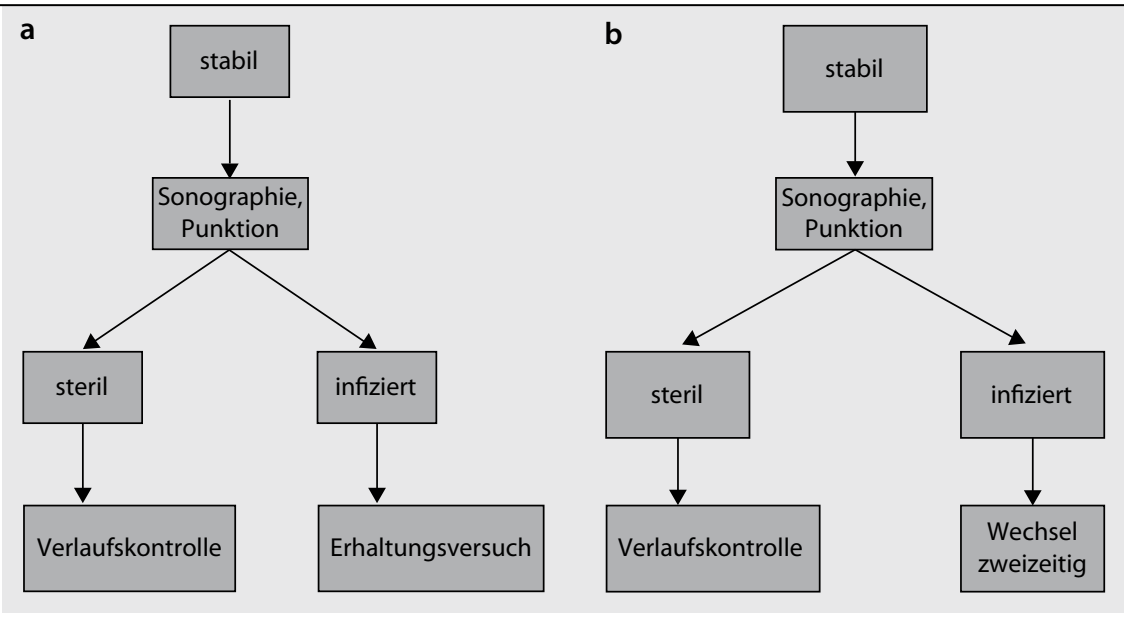

eine individuelle Therapie eingeleitet. In allen Fällen orientiert sich das therapeutische Vorgehen an den in $\mathbf{A b b} \mathbf{3}$ grafisch aufgeführten Algorithmen.

Definitionsgemäß wird beim Auftreten eines Infektes 3-4 Wochen nach Primärimplantation der Prothese von einem Frühinfekt gesprochen. In diesem Zeitraum befinden sich die Bakterien noch in einer planktonischen Phase und sind einer lokalen und systemischen Antibiotikatherapie gut zugänglich [23].

Wird der Zeitraum von 3-4 Wochen überschritten (Spätinfekt), kommt es zunehmend, zumindest bei Schleimbildnern, zur Ausbildung einer gut geschützten sessilen Phase. In dieser liegen die Bakterien eingebettet in einer Schleimmatrix auf den Prothesenoberflächen und sind mit dieser fest verbunden. Eine Befundrevision mit ausgedehntem Débridement und eine systemische Antibiotikatherapie sind in diesem Fall zur Infektsanierung nicht mehr ausreichend, sondern es sollte eine Prothesenexplantation erfolgen. Ergeben sich präoperativ oder intraoperativ Hinweise für eine Prothesenlockerung, ist diese ebenfalls zu explantieren. Die Prothesenwechsel werden in unserer Abteilung bei nachgewiesenem Infekt immer zweizeitig vorgenommen.

\section{Analyse}

In einer retrospektiven Untersuchung überprüften wir die in unserer Abteilung durchgeführten Punktionen von Hüftund Kniegelenken bei klinischem Verdacht auf das Vorliegen eines periprothetischen Infektes.

Viele unserer Patienten wurden bereits in anderen Krankenhäusern, bei mikrobiologisch nachgewiesenem Infekt, mehrfach voroperiert. Bei einer anderen großen Patientengruppe lag bereits ein positives Ergebnis einer auswärts durchgeführten Punktion vor. Aus diesen Gründen fanden sich im eigenen Patientengut, trotz Ausdehnung des Untersuchungszeitraumes auf 7 Jahre, nur 29 eigene Hüftund 34 eigene Kniegelenkpunktionen. Eine sinnvolle statistische Aufarbeitung war somit nicht möglich. Die dargestellten Zahlen sollen jedoch einen Überblick über die gewonnenen Ergebnisse ermöglichen.

Abb. $3 \Delta$ Therapieschema bei a Früh- und b Spätinfekt

Das hier beschriebene Dilemma erklärt z. T. die erhebliche Bandbreite der in der Literatur angegebenen Zahlen für die Sensitivität und Spezifität für diese Diagnostikmethode, obgleich sie weiterhin als „Goldstandard“ bezeichnet wird.

Hüfte. Im Zeitraum vom 23.05.200125.06.2008 wurden in unserer Abteilung 29 Hüftgelenkpunktionen bei Verdacht auf einen periprothetischen Infekt durchgeführt. In $45 \%$ fand sich ein positives Keimergebnis. Entsprechend des oben beschriebenen Therapiealgorithmus schloss sich in allen Fällen eine operative Revision an. In 55\% der Fälle gelang ein Keimnachweis im untersuchten Punktat nicht. Bei führender Klinik wurde die Hälfte der Patienten mit negativem Keimbefund ebenfalls revidiert, die andere Hälfte wurde klinisch beobachtet und zeigte einen unauffälligen weiteren Verlauf.

Die Gruppe der revidierten Hüften mit positivem Punktionsergebnis wies nach intraoperativer Entnahme einer Gewebeprobe in $77 \%$ der Fälle einen mit dem Punktionsergebnis identischen Keim auf. In 23\% zeigte sich ein Keimwechsel. Die Gruppe der revidierten Hüften mit negativem Punktionsergebnis bei führender Klinik zeigte in $62,5 \%$ einen positiven, mit dem Punktionsergebnis übereinstimmenden Keimbefund der untersuchten Gewebeprobe. In 37,5\% konnte auch anhand der Gewebeproben, bei klinisch sicherem Infekt, kein Keim nachgewiesen werden.

Knie. Sehr ähnliche Ergebnisse wie für die Hüfte fanden sich für die 34 im Un- tersuchungszeitraum vom 16.01.200225.06.2008 durchgeführten Kniegelenkpunktionen bei Verdacht auf einen periprothetischen Infekt. 59\% wiesen einen positiven Keimbefund auf. In diesen Fällen schloss sich, wie bei den Hüftgelenken, eine Revision an. In $41 \%$ konnte kein Keim isoliert werden. In dieser Gruppe wurde die Hälfte der Patienten, aufgrund der führenden Klinik, einer Revision mit Entnahme einer Gewebeprobe zugeführt. Die andere Hälfte (20,5\%) wurde nachbeobachtet und blieb, betreffend eines Infektes, klinisch weiter stumm.

In der Gruppe der revidierten Kniegelenke mit vorangegangenem negativem Punktionsergebnis fand sich nach Untersuchung einer Gewebeprobe in $71,5 \%$ ein positiver Keimbefund. In den anderen Fällen blieb die untersuchte Gewebeprobe steril. Die Gruppe der revidierten Kniegelenke mit vorangegangenem positivem mikrobiologischem Befund wies in $80 \%$ nach der Entnahme einer Gewebeprobe den gleichen Keim mit gleichem Antibiogramm auf. In 5\% konnte ein Keimwechsel beobachtet werden. In 15\% der Fälle gelang anhand der Gewebeprobe kein Erregernachweis mehr, und die Proben blieben steril.

\section{Diskussion}

In der septischen Chirurgie ist der Evidenzgrad der vorhandenen Studien deutlich geringer als z. B. in der Onkologie oder der Kardiologie. Der Hauptgrund hierfür ist eine geringe Fallzahl von vergleichbaren Patienten. Das Patientenspektrum in der septischen Unfallchir- 


\section{Diagnostik der Osteitis}

urgie weist eine große Heterogenität mit einer Vielzahl von verschiedenen begleitenden Erkrankungen und Risikofaktoren auf. Ein Mangel an belastbarer Evidenz ist jedoch nicht gleichzusetzen mit einer Unwirksamkeit der verschiedenen eingesetzten diagnostischen und therapeutischen Strategien. Bisher ist jedoch unklar, welche der vielen wirksamen Strategien in welchem Fall einer anderen überlegen ist. Die Lösung dieses Problems scheint nur in mehreren Schritten möglich zu sein und setzt die Zusammenarbeit verschiedener, auf diesem Fachgebiet arbeitender Zentren voraus.

Im ersten Schritt scheint es notwendig, in der septischen Chirurgie einen einheitlichen Sprachgebrauch zu finden und die verschiedenen Begrifflichkeiten klar und eindeutig zu definieren. So ist der Begriff der Osteitis bis heute nur unscharf definiert und zu anderen Infektionen mit Beteiligung des Knochens nicht klar abgegrenzt.

In einem zweiten Schritt könnten durch klinikübergreifende retrospektive Studien verschiedene diagnostische und therapeutische Strategien in ihrer Wirksamkeit überprüft und verglichen werden.

Durch randomisierte prospektive Multicenterstudien können dann, in einem dritten Schritt, Aussagen mit hohem Evidenzgrad gemacht werden.

Um dieses Ziel zu erreichen, wurde vor einigen Monaten die Arbeitsgemeinschaft Septische Chirurgie gegründet.

\section{Korrespondenzadresse}

\section{W. Hirschberger}

BG-Unfallklinik Frankfurt am Main,

Friedberger Landstraße 430,

60389 Frankfurt am Main

whirschberger@aol.com

Interessenkonflikt. Der korrespondierende Autor gibt an, dass kein Interessenkonflikt besteht.

\section{Literatur}

1. Antes G, Diener MK (2006) The role of systematic reviews in evidence-based healthcare. Chin J Evid Based Med 6:467-470

2. Antes G, Sauerland S, Seiler CM (2006) Evidencebased medicine - from best research evidence to a better surgical practice and health care. Langenbecks Arch Surg 391:61-67
3. Atkins BL, Athanasou N, Deeks JJ (1998) Prospective evaluation of criteria for microbiological diagnosis of prothetic-joint infection at hip arthroplasty. The OSIRIS Collaborative Study Group. J Clin Microbiol 36:2932-2939

4. Barrack RL, Jennings RW, Wolfe MW, Bertot AJ (1997) The Coventry Award. The value of preoperative aspiration before total knee revision. Clin Orthop 345:8-16

5. Bauer H (2005) Evidenzbasierte Medizin. Chirurg 76:795-796

6. Cochrane A (1972) Effectiveness and efficiency: random reflections on health services. Nuffield Provincial Hospital Trust, London

7. Diener MK, Seiler CM, Antes G (2007) Systematische Übersichtsarbeiten und Metaanalysen in der Chirurgie. Chirurg 78:938-944

8. Dixon E, Hameed M, Sutherland F et al (2005) Evaluating meta-analyses in the general surgical literature: a critical appraisal. Ann Surg 241:450-459

9. Duff GP, Lachiewics PF, Kelly SS (1996) Aspiration of the knee joint before revision arthroplasty. Clin Orthop 331:132-139

10. Egger M, Smith GD, Altman DG (2001) Systematic reviews in health care: meta-analysis in context. BMJ Publishing Group, London

11. Eisler T, Svensson O, Engstrom CF et al (2001) UItrasound for diagnosis of infection in revision total hip arthroplasty. J Arthroplasty 16:1010-1017

12. Fuerst $M$, Fink $B$, Rüther W (2005) Die Wertigkeit von präoperativer Punktion und arthroskopischer Synovialisprobenentnahme bei Knietotalendoprothesenwechsel. Z Orthop Ihre Grenzgeb 143:3642

13. Frommelt $L$ (2008) Gelenkpunktat und Erregernachweis bei periprothetischer Infektion. Orthopäde 37:1027-1036

14. Haaker R, Senge A, Krämer J, Rubenthaler F (2004) Osteomyelitis nach Endoprothesen. Orthopäde 33:431-438

15. Lange V (2003) Evidence-based Medicine im chirurgische Alltag - Anspruch und Wirklichkeit. Mitt Dtsch Ges Chir 32:143-146

16. Lüring C, Koester B, Grifka J (2006) Gibt es Evidenz in der evidenzbasierten Medizin? Z Orthop Ihre Grenzgeb 144:563-568

17. Mulrow CD (1994) Rationale for systematic reviews. BMJ 309:597-599

18. Sackett DL, Rosenberg WM, Gray JA et al (1996) Evidence based medicine: what it is and what it isn't. BMJ 312:71-72

19. Sauerland S, Seiler CM (2005) Role of systematic reviews and meta-analysis in evidence-based medicine. World J Surg 29:582-587

20. Sawicki PT (2005) Evidenzbasierte Medizin. Keine „Zwangsjacke" für den Arzt. Dtsch Ärztebl 102:A 888-892

21. Steinbrink K, Frommelt L (1995) Behandlung der periprothetischen Infektion der Hüfte durch einzeitige Austauschoperation. Orthopäde 24:335343

22. Trampuz A, Hanssen AD, Osmon DR et al (2004) Synovial fluid leukocyte count and differential for the diagnosis of prosthetic knee infection. Am J Med 117:556-562

23. Tsukayama DT, Estrada R, Gustillo RB (1996) Infection after total hip arthroplasty. A study of the treatment of one hundred and six infections. J Bone Joint Surg Am 78:512-523

24. Virolainen $P$, Lahteenmaki $H$, Hiltunen $A$ (2002) The reliability of diagnosis of infection during revision arthroplasties. Scand J Surg 91:178-181 